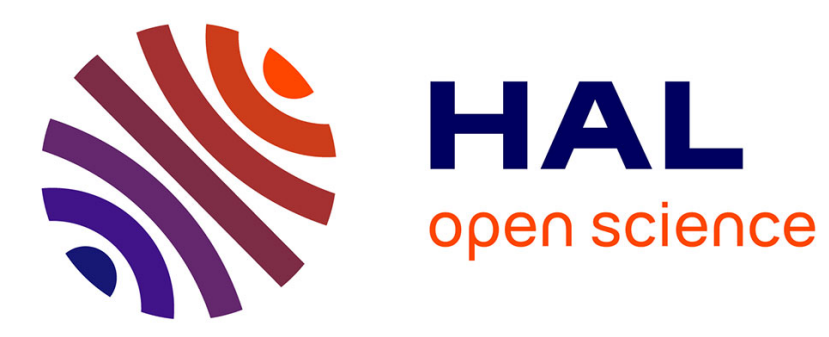

\title{
Milieux en couches et ondes dispersives
}

\author{
F. Léomy, M. de Billy, G. Quentin
}

\section{To cite this version:}

F. Léomy, M. de Billy, G. Quentin. Milieux en couches et ondes dispersives. Revue de Physique Appliquée, 1988, 23 (9), pp.1547-1555. 10.1051/rphysap:019880023090154700 . jpa-00245983

\section{HAL Id: jpa-00245983 https://hal.science/jpa-00245983}

Submitted on 1 Jan 1988

HAL is a multi-disciplinary open access archive for the deposit and dissemination of scientific research documents, whether they are published or not. The documents may come from teaching and research institutions in France or abroad, or from public or private research centers.
L'archive ouverte pluridisciplinaire HAL, est destinée au dépôt et à la diffusion de documents scientifiques de niveau recherche, publiés ou non, émanant des établissements d'enseignement et de recherche français ou étrangers, des laboratoires publics ou privés. 
Classification

Physics Abstracts

$43.30-43.35-68.45 \mathrm{~K}-68.35 \mathrm{~J}$

\title{
Milieux en couches et ondes dispersives
}

\author{
F. Léomy, M. de Billy et G. Quentin \\ Groupe de Physique des Solides, Université Paris 7, Tour 23, 2 place Jussieu, 75251 Paris Cedex 05, France
}

(Reçu le 28 mars 1988, révisé le 26 mai 1988, accepté le 2 juin 1988)

\begin{abstract}
Résumé. - Lorsqu'un faisceau acoustique borné est réfléchi par un solide semi-infini ou une plaque immergée, on observe pour certains angles d'incidence des effets non spéculaires. A partir de mesures de réflectivité, nous avons établi les courbes de dispersion des vitesses de phase des ondes dispersives (de type Lamb, Rayleigh ou Sezawa) générées dans des échantillons présentant deux types de configuration : une configuration bilame incluant deux plaques métalliques de faible épaisseur collées ou soudées et une configuration constituée d'un milieu solide semi-infini (substrat) chargé par une plaque soudée ou collée. Les matériaux retenus pour cette étude sont l'étain, le laiton et le duraluminium. Les courbes expérimentales obtenues sont discutées et comparées aux prévisions théoriques.
\end{abstract}

\begin{abstract}
Non specular effects are observed when an ultrasonic bounded beam is incident on a layered medium immersed in water. These reflectivity measurements are used to obtain the phase velocity dispersion curves of the « leaky » surface acoustic waves which are generated in the layered specimen. Two configurations with different boundary conditions are investigated : a bilaminar system including two welded or two bonded layers and a semi-infinite elastic medium loaded with a welded or a bonded layer. The various materials used in the samples are : tin, brass and duraluminum. The experimental results are discussed and compared with the theoretical computations.
\end{abstract}

\section{Introduction.}

Il est maintenant bien établi que des phénomènes non spéculaires se produisent lors de la réflexion d'un faisceau acoustique borné à une interface plane séparant un milieu fluide et un milieu solide élastique semi-infini [1-5]. Parmi ces effets, la présence d'un minimum dans le coefficient de réflexion révèle l'existence d'une onde de surface de type Rayleigh qui rayonne continûment dans le milieu fluide au cours de sa propagation. Dans le cas où l'échantillon insoné est une plaque de faible épaisseur (comparée à la longueur d'onde), des phénomènes analogues se produisent chaque fois que l'angle d'incidence permet la génération d'une onde de type Lamb à l'intérieur de la plaque [6-8]. Comme ces ondes sont dispersives, la position des minima (reliée à la vitesse de phase par la loi de Descartes) évolue avec la fréquence. Ainsi, la mesure des fréquences de résonances (à angle fixe) ou des angles critiques (à fréquence fixe) permet d'établir les courbes de dispersion des différents modes de plaques.

Des études récentes ont été menées sur des échantillons constitués d'un substrat chargé par une fine couche [9-15] et pour lesquels des phénomènes analogues à ceux mentionnés précédemment ont été observés. Ces études ont révélé que suivant la nature du couplage, les conditions aux limites changent entraînant dans certains cas une modification très importante des courbes de dispersion. Nous nous proposons de faire une analyse comparative des courbes de dispersion obtenues dans le cas de deux configurations particulières :

- une configuration bilame constituée de deux plaques métalliques soudées ou collées ;

- une configuration comprenant un milieu solide semi-infini (ou substrat) chargée par une plaque métallique de faible épaisseur collée ou soudée.

Si cette étude, basée sur des mesures de réflectivité et d'analyse fréquentielle confirme certains résultats expérimentaux déjà rapportés à ce jour, elle apporte aussi des éléments supplémentaires sur le caractère « rayonnant » des ondes de Sezawa et de Rayleigh et devrait permettre de tester les modèles théoriques élaborés pour ce type d'échantillons [16-20]. 
1. Dispositif de mesure et procédure expérimentale.

Deux méthodes complémentaires ont été développées pour établir les courbes de dispersion représentatives des variations de la vitesse de phase (ou de l'angle d'incidence) en fonction du produit $F d$ ( $F$ représente la fréquence d'excitation du capteur et $d$ l'épaisseur de la plaque. Rappelons que l'angle d'incidence $\theta_{\mathrm{i}}$ et la vitesse de phase $V_{\phi}$ de l'onde sont liés par la relation de Snell-Descartes : $\sin \theta_{i}=$ $V_{\text {fluide }} / V_{\phi}$. La première de ces méthodes consiste à faire, à fréquence fixe, une analyse angulaire de l'amplitude du signal spéculairement réfléchi et à relever les angles critiques pour lesquels on observe un minimum. La deuxième méthode est fondée sur une analyse spectrale du signal spéculairement diffusé ; les minima observés dans chaque spectre indiquent la valeur des fréquences d'excitation des modes propres de l'échantillon.

Le dispositif de mesurè est représenté sur la figure 1 ; il comprend un banc de mesure automatisé, une sonde émettrice fixe et une sonde réceptrice mobile animée d'un mouvement de rotation autour du même axe que la cible. Le signal détecté après réflexion sur la face plane de l'échantillon est amplifié puis visualisé sur l'écran d'un oscilloscope de contrôle avant d'être échantillonné en vue de traitements ultérieurs (analyse fréquentielle, moyenne, etc...). L'utilisation de palpeurs spécifiques à la méthode d'analyse est nécessaire de même que leur condition d'excitation : impulsion courte pour les sondes large-bande (analyse fréquentielle), impulsion longue pour les sondes à bande-étroite (analyse angulaire). Des palpeurs répondant à des fréquences centrales différentes ont été utilisés de façon à couvrir la gamme de fréquence : 0,8$10 \mathrm{MHz}$. Les variations du produit $F d$ sont donc assurées par une modification soit de la fréquence d'excitation, soit de l'épaisseur de la plaque suivant le type d'échantillon. Les limites d'exploration angulaires sont définies par l'encombrement du support mécanique des capteurs : $10^{\circ}-70^{\circ}$. La face insonée de chacun des échantillons contient l'axe de rotation de la cible et de la sonde réceptrice. L'ensemble est immergé dans une cuve remplie d'eau $\left(V_{\text {fluide }}=\right.$ $1480 \mathrm{~m} / \mathrm{s}$ ).

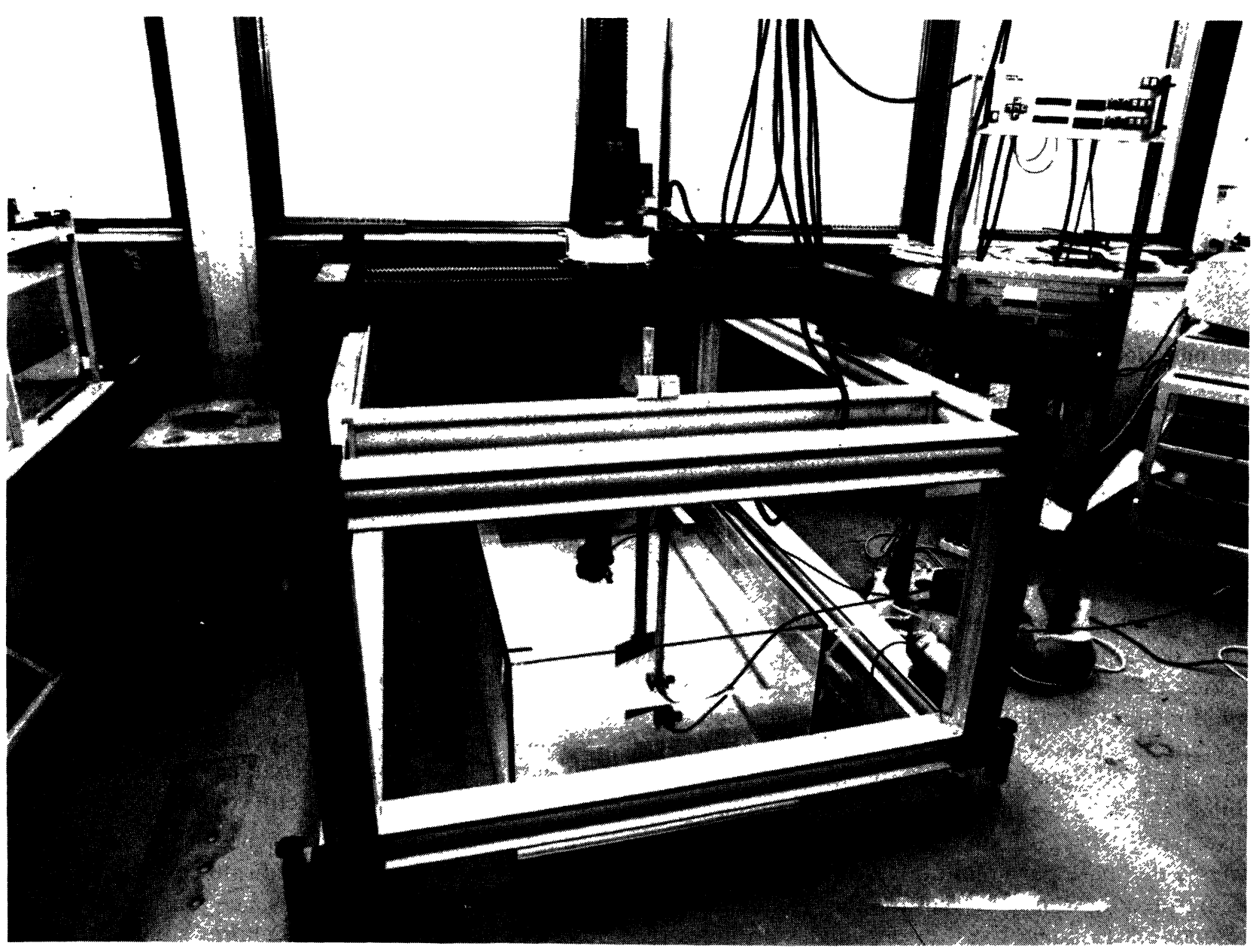

Fig. 1. - Photographie du dispositif expérimental.

[Photograph of the experimental equipment.] 


\section{Résultats expérimentaux.}

Les courbes de dispersion de la vitesse de phase ont été obtenues avec quatre échantillons dont les caractéristiques sont rappelées dans le tableau I et dont le choix se justifie par l'objectif initial de cette étude qui est le contrôle des collages par voie ultrasonore.

2.1 DESCRIPTION DES ÉCHANTILLONS. - Les différentes structures des échantillons étudiées sont schématisées sur la figure 2 . Les échantillons soudés ont été réalisés en fondant une couche d'étain sur un substrat ou une plaque de laiton. Nous désignerons respectivement par $E S_{\mathrm{ps}}^{\mathrm{e} \ell}$ et $E S_{\mathrm{pp}}^{\mathrm{e} \ell}$ les deux échantillons analysés. Les indices inférieur et supérieur gauches caractérisent le premier milieu rencontré $(\mathrm{e}=$ étain et $\mathrm{p}=$ plaque), les deux autres indices définissent le deuxième milieu rencontré $(\ell=$ laiton et $\mathrm{s}=$ substrat ou milieu semi-infini). De façon similaire, nous avons défini les échantillons $\mathrm{EC}_{\mathrm{ps}}^{\ell_{\mathrm{d}}}$ et $\mathrm{EC}_{\mathrm{pp}}^{\ell_{\mathrm{d}}}$ constitués de deux éléments métalliques collés avec une fine couche d'Araldite ou de couplant «Sofranel» de $200 \mu \mathrm{m}$ environ.

Une analyse du tableau I montre que dans le cas d'échantillons constitués de deux plaques le rapport entre les épaisseurs des deux lames (collées ou soudées) est de l'ordre de l'unité. Selon les conditions expérimentales (fréquences, échantillons) on note que la longueur d'onde dans le milieu incident (eau) est inférieure ou de l'ordre de l'épaisseur de la plaque.

Sur le tableau II, sont données les caractéristiques acoustiques des divers matériaux utilisés. Soulignons que la vitesse de l'onde de cisaillement $\left(C_{\mathrm{T}}\right)$ dans l'étain est inférieure à celle de l'eau, ce qui justifie l'absence d'angle critique $\theta_{\mathrm{T}}$ pour ce matériau.

2.2 RÉSUlTATS. - Sur la figure 3 nous donnons des exemples typiques de coefficient de réflexion obtenus avec les échantillons décrits précédemment. La figure 4 , quant à elle, est représentative des spectres

Tableau I. - Tableau descriptif des échantillons étudiés.

[Characteristics of the samples.]

\begin{tabular}{|c|c|c|c|c|c|}
\hline Echantillon & & $\begin{array}{l}\text { Nature de la plaque } \\
\text { ou de la couche }\end{array}$ & $\begin{array}{l}\text { Nature } \\
\text { ou de }\end{array}$ & $\begin{array}{l}\text { du substrat } \\
\text { la plaque }\end{array}$ & Nature du contact \\
\hline $\begin{array}{l}\mathrm{EC}_{\mathrm{ps}}^{\ell \mathrm{d}} \\
\mathrm{EC}_{\mathrm{pp}}^{\ell \mathrm{d}} \\
\mathrm{ES}_{\mathrm{ps}}^{\mathrm{el}} \\
\mathrm{ES}_{\mathrm{pp}}^{\mathrm{el}}\end{array}$ & $\begin{array}{l}\text { laiton } \\
\text { laiton } \\
\text { étain } \\
\text { étain }\end{array}$ & $\begin{array}{l}(d=1,7 \mathrm{~mm}) \\
\left(d_{1}=0,81 \mathrm{~mm}\right) \\
\left(d_{1}=1 ; 0,38 ; 0,22 \mathrm{~mm}\right) \\
(d=1,02 \mathrm{~mm})\end{array}$ & $\begin{array}{l}\text { duraluminium } \\
\text { duraluminium } \\
\text { laiton } \\
\text { laiton }\end{array}$ & $\begin{array}{l}\left(d^{\prime}=25,0 \mathrm{~mm}\right) \\
\left(d_{2}=15,01 \mathrm{~mm}\right) \\
\left(d_{2}=25,0 \mathrm{~mm}\right) \\
\left(d^{\prime}=0,99 \mathrm{~mm}\right)\end{array}$ & $\begin{array}{cc}\text { collage } & (e=0,19 \mathrm{~mm}) \\
\text { collage } & (e=0,17 \mathrm{~mm}) \\
\text { soudure } & \\
\text { soudure } & \end{array}$ \\
\hline
\end{tabular}
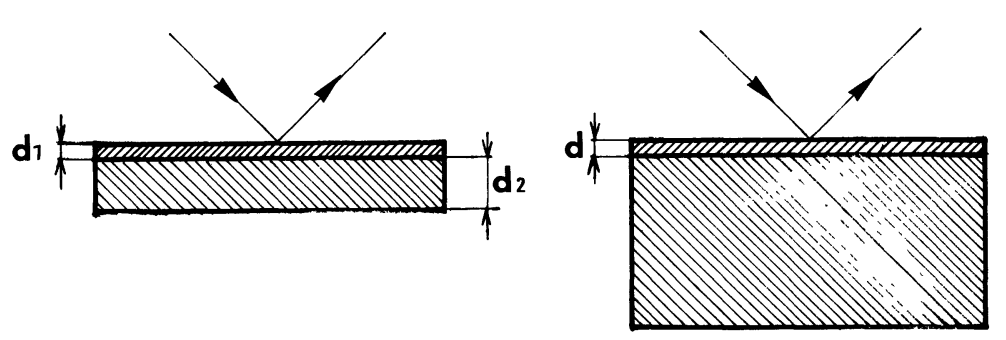

c

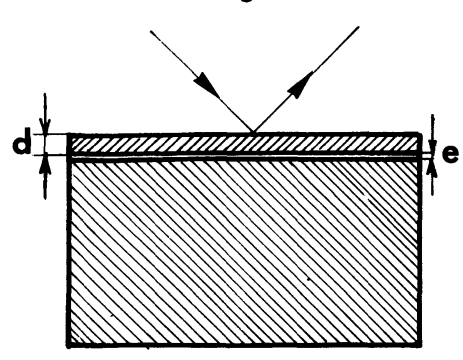

d

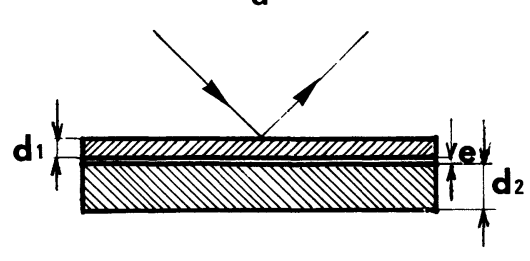

Fig. 2. - Schémas des échantillons étudiés.

[Schematic cross-section of the samples.] 
Tableau II. - Propriétés acoustiques des matériaux.

[Acoustical properties of the materials.]

\begin{tabular}{|lc|c|c|c|c|}
\hline & $\begin{array}{c}C_{\mathrm{L}} \\
(\mathrm{m} / \mathrm{s})\end{array}$ & $\begin{array}{c}\theta_{\mathrm{L}} \\
(\mathrm{deg})\end{array}$ & $\begin{array}{c}C_{\mathrm{T}} \\
(\mathrm{m} / \mathrm{s})\end{array}$ & $\begin{array}{c}\theta_{\mathrm{T}} \\
(\mathrm{deg})\end{array}$ & $\begin{array}{c}\rho \times 10^{3} \\
\left(\mathrm{~kg} / \mathrm{m}^{3}\right)\end{array}$ \\
\hline $\begin{array}{l}\text { Duraluminium } \\
\text { Laiton }\end{array}$ & 6286 & 13,6 & 3278 & 26,8 & 2,8 \\
Etain & 4480 & 19,3 & 2156 & 43,3 & 8,1 \\
Eau & 2930 & 30,3 & 1300 & - & 8,5 \\
$\begin{array}{l}\text { Araldite } \\
\text { gel « Sofranel » }\end{array}$ & 1480 & - & - & - & 1 \\
\hline
\end{tabular}
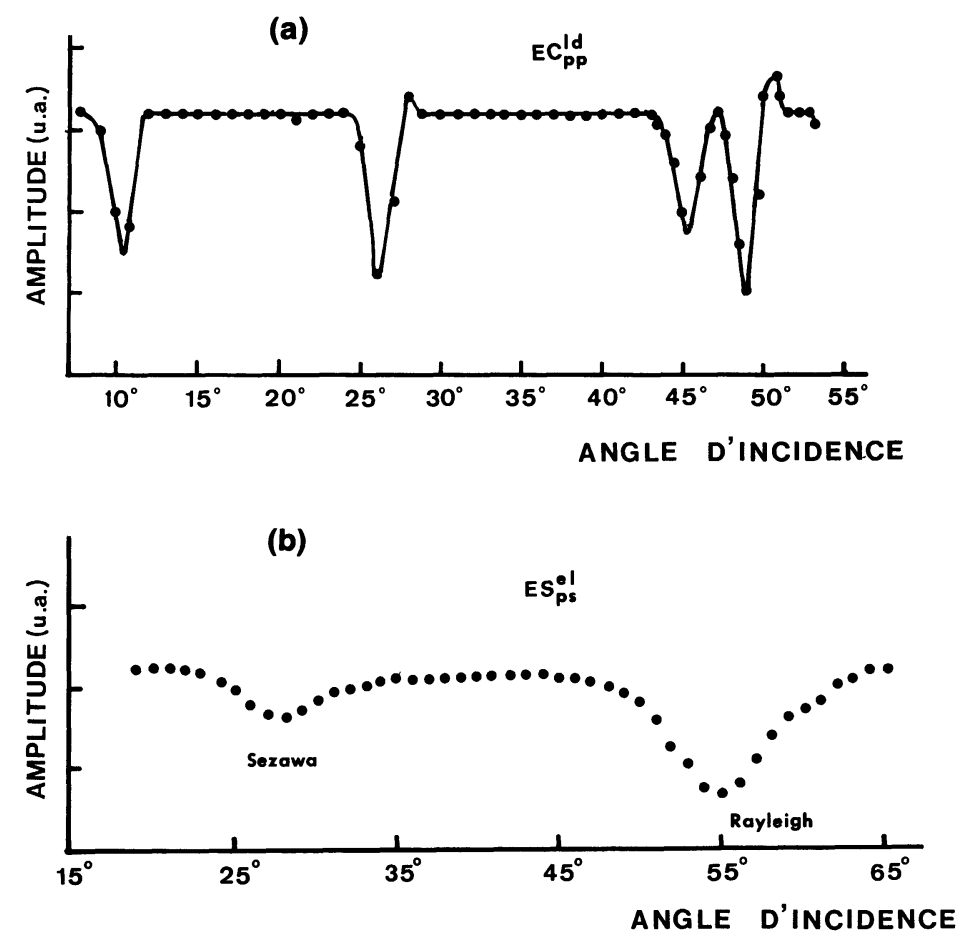

(c)

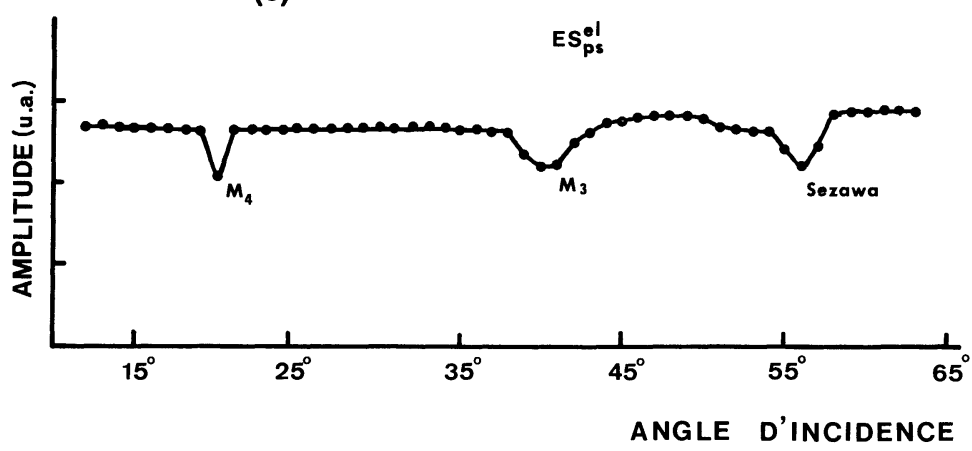

Fig. 3. - Exemples typiques des variations angulaires expérimentales de l'amplitude du signal réfléchi spéculairement : (a) échantillon $\mathrm{EC}_{\mathrm{pp}}^{\ell \mathrm{d}}(F d=2,66 \mathrm{MHz} \mathrm{mm}) ;$ (b) échantillon $\mathrm{ES}_{\mathrm{ps}}^{\mathrm{el}}(F d=0,18 \mathrm{MHz} \mathrm{mm})$; (c) échantillon $\mathrm{ES}_{\mathrm{ps}}^{\mathrm{el}}$ $(F d=1,2 \mathrm{MHz} \mathrm{mm})$.

[Typical examples of experimental reflection coefficient with an arbitrary amplitude scale : (a) sample $\mathrm{EC}_{\mathrm{pp}}^{\ell \mathrm{d}}$ $(F d=2.66 \mathrm{MHz} \mathrm{mm}) ;\left(\right.$ b) sample $\operatorname{ES}_{\mathrm{ps}}^{\mathrm{el}}(F d=0.18 \mathrm{MHz} \mathrm{mm}) ;\left(\right.$ c) sample $\mathrm{ES}_{\mathrm{ps}}^{\mathrm{el}}(F d=1.2 \mathrm{MHz} \mathrm{mm})$.] 


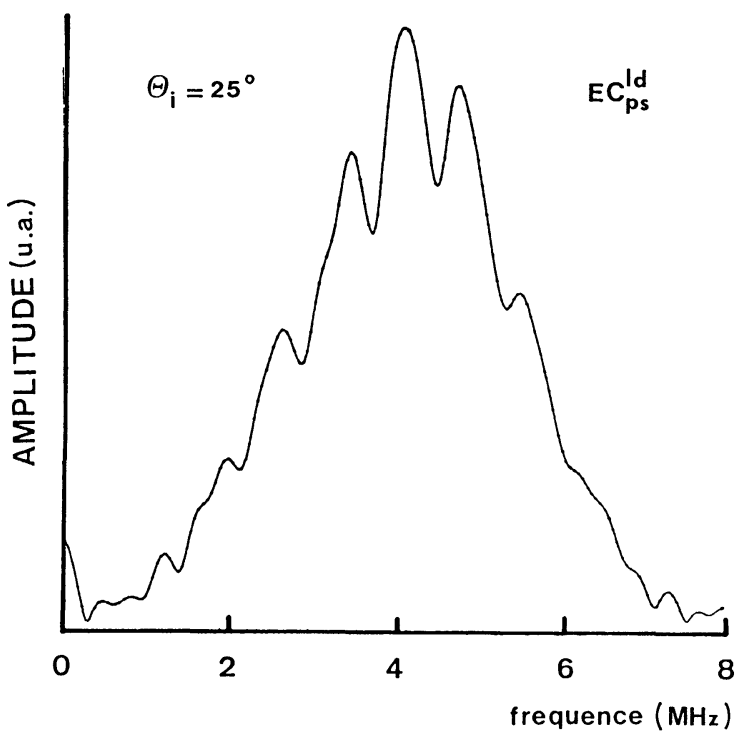

Fig. 4. - Exemple typique d'un spectre de fréquence obtenu à $\theta_{\mathrm{i}}=25^{\circ}$ avec l'échantillon $\mathrm{EC}_{\mathrm{ps}}^{\ell \mathrm{d}}$.

[Typical example of a spectrum at $\theta_{\mathrm{i}}=25^{\circ}$ obtained with sample $\mathrm{EC}_{\mathrm{ps}}^{\ell_{\mathrm{d}}}$.]

de fréquences observés lors des études en analyse fréquentielle.

Sur les figures suivantes (Figs. 5 à 8) sont tracées les courbes de dispersion expérimentales obtenues à partir des analyses angulaires ou fréquentielles. Elles représentent les variations de l'angle d'incidence, associées aux variations de la vitesse de phase, en fonction du produit $F d$, pour les divers échantillons sélectionnés.

Echantillon $\mathrm{EC}_{\mathrm{ps}}^{l_{\mathrm{d}}}$. - Les points expérimentaux sont représentés sur la figure 5. L'épaisseur de la couche de couplant est estimée à $0,2 \mathrm{~mm}$. Des mesures effectuées en fonction de l'épaisseur de colle $(d=0,1 \mathrm{~mm}$ et $0,05 \mathrm{~mm})$ n'ont révélé aucune différence dans les courbes de dispersion pour des angles supérieurs à l'angle critique longitudinal du duraluminium $\left(\theta_{L}=19,3^{\circ}\right)$. Pour des angles d'incidence inférieurs à $20^{\circ}$ environ la répartition des points semble en revanche dépendre légèrement de l'épaisseur du couplant tout particulièrement à basse fréquence.

Echantillon $\mathrm{EC}_{\mathrm{pp}}^{\mathrm{ld}_{\mathrm{d}}}$ - - Les courbes de dispersion relatives aux différents modes dans le cas de deux plaques métalliques collées à l'araldite sont tracées sur les figures $6 \mathrm{a}$ et $6 \mathrm{~b}$. Elles correspondent respectivement à une incidence sur la plaque de laiton et sur la plaque de duraluminium. Nous avons porté en abscisse le produit de la fréquence par l'épaisseur de la première plaque recevant le faisceau acoustique.

Echantillon $\mathrm{ES}_{\mathrm{ps}}^{\mathrm{e} \ell}$ - - La figure 7a rend compte des courbes de dispersion de l'échantillon constitué d'une plaque d'étain soudée à un substrat de laiton. Les courbes sont limitées aux faibles valeurs du produit $F d$ ( $d$ représente ici l'épaisseur de la plaque d'étain). La figure $7 \mathrm{~b}$ représente les variations de la vitesse de phase des différents modes pour des valeurs plus élevées de la variable $F d$.

Echantillon $\mathrm{ES}_{\mathrm{pp}}^{\mathrm{el}}$. - Dans le cas de l'échantillon constitué de deux plaques soudées, les courbes de dispersion mesurées expérimentalement sont indiquées sur la figure 8. Elles correspondent aux différents modes propres de vibration de l'échantillon, assimilé à une plaque unique. Ces courbes sont observées quel que soit le matériau constituant la première plaque rencontrée par le faisceau incident. Enfin, ces résultats obtenus en réflexion sont confirmés par une analyse en transmission.

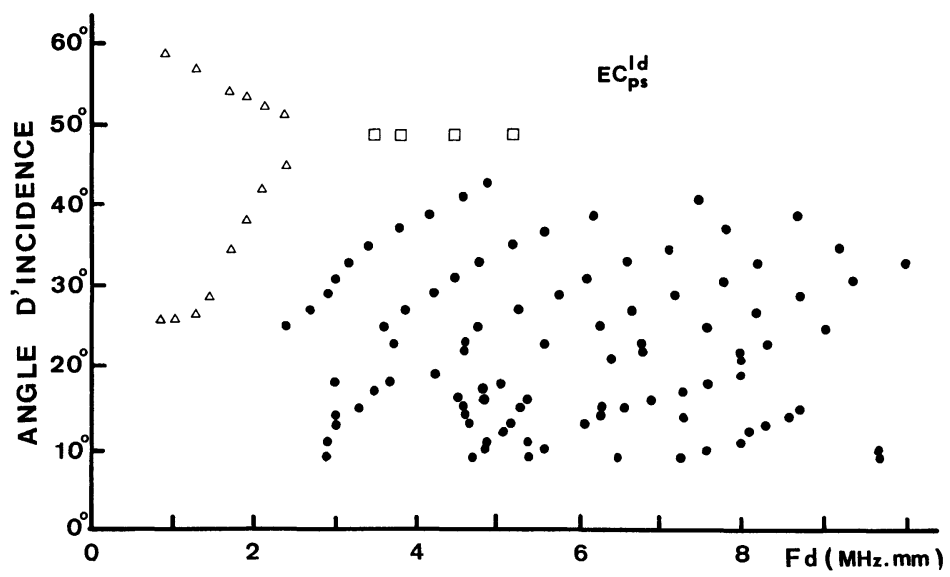

Fig. 5. - Courbes de dispersion obtenues pour l'échantillon $\mathrm{EC}_{\mathrm{ps}}^{l_{\mathrm{d}}}:(\square)$ analyse angulaire (Fréquence centrale $=$ $2,25 \mathrm{MHz}) ;(\Delta)$ analyse angulaire (Fréquence centrale $=1 \mathrm{MHz}) ;(\bullet)$ analyse spectrale.

[Dispersion curves for sample $\mathrm{EC}_{\mathrm{ps}}^{\ell_{\mathrm{d}}}:(\square)$ angular analysis (Central frequency $\left.=2.25 \mathrm{MHz}\right) ;(\triangle)$ angular analysis (Central frequency $=1 \mathrm{MHz}) ;(\bullet)$ spectral analysis.] 

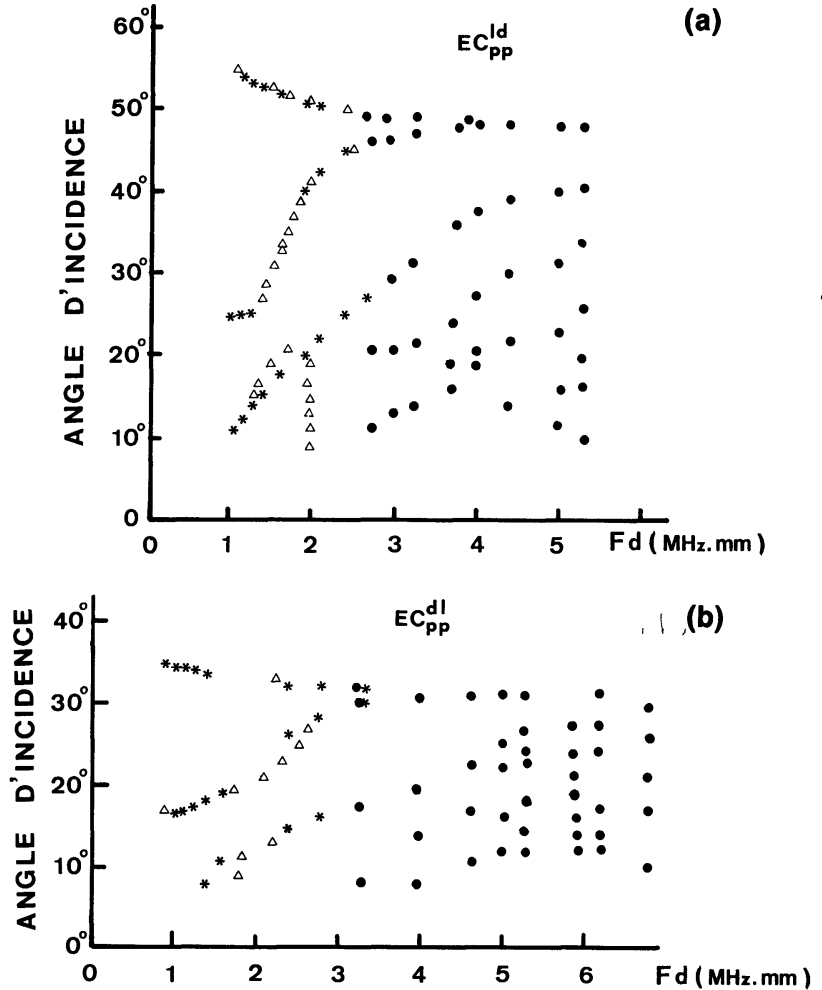

Fig. 6. - Courbes de dispersion obtenues pour l'échantillon constitué de deux plaques collées (épaisseur de la plaque de laiton $d_{1}=0,81 \mathrm{~mm}$; épaisseur de la plaque de duraluminium $\left.d_{2}=1,01 \mathrm{~mm}\right)$. Pour chaque courbe : $(\triangle)$ analyse spectrale ; $(\bullet)$ analyse angulaire (Fréquence centrale $=5 \mathrm{MHz}) ;(*)$ analyse angulaire (Fréquence centrale $=2,25 \mathrm{MHz}$ ). (a) Incidence sur la plaque de laiton $\left(\mathrm{EC}_{\mathrm{pp}}^{\ell \mathrm{d}}\right)$; (b) incidence sur la plaque de duraluminium $\left(\mathrm{EC}_{\mathrm{pp}}^{\mathrm{d} \ell}\right)$.

[Dispersion curves for the sample made of two plates glued (thickness of brass plate $d_{1}=0.81 \mathrm{~mm}$; thickness of duraluminum plate $\left.d_{2}=1.01 \mathrm{~mm}\right)$. For each curve : $(\triangle)$ spectral analysis; $(\bullet)$ angular analysis (Central frequency $=5 \mathrm{MHz}$ ); $(*)$ angular analysis (Central frequency $=2.25 \mathrm{MHz}$ ). (a) Incidence on the brass plate $\left(E C_{\mathrm{pp}}^{\ell \mathrm{d}}\right) ;(\mathrm{b})$ incidence on the duraluminum plate $\left(\mathrm{EC}_{\mathrm{pp}}^{\mathrm{d} \ell}\right)$.]

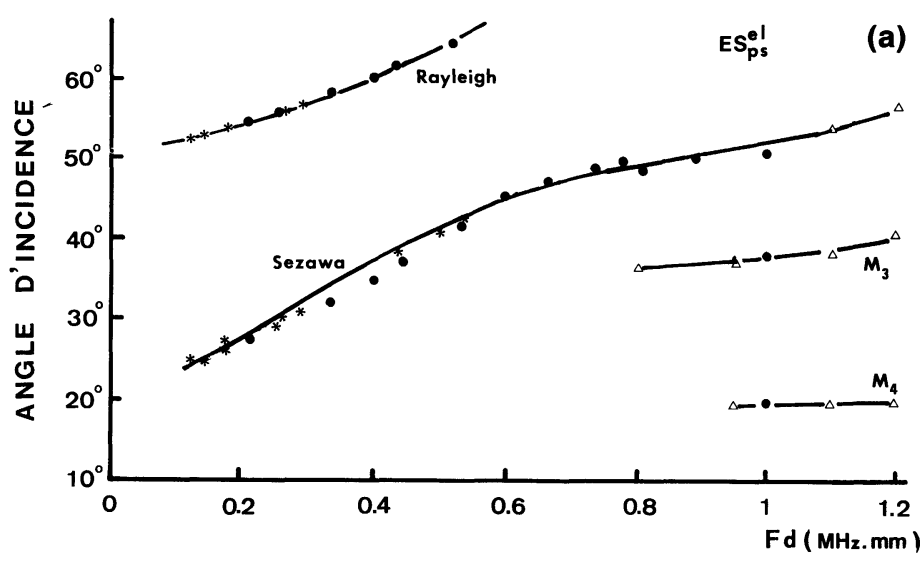

Fig. 7. - (a) Courbes de dispersion obtenues pour l'échantillon $\mathrm{ES}_{\mathrm{ps}}^{\mathrm{e}}$. $(0<F d<1,2)$. (b) Courbes de dispersion obtenues pour l'échantillon $\operatorname{ES}_{\mathrm{ps}}^{\mathrm{el}}(0<F d<2,5)$.

[Dispersion curves observed for sample $\mathrm{ES}_{\mathrm{ps}}^{\mathrm{el}}(0<F d<1,2)$. (b) Dispersion curves observed for sample $\mathrm{ES}_{\mathrm{ps}}^{\mathrm{e}}$ $(0<F d<2,5)$.]
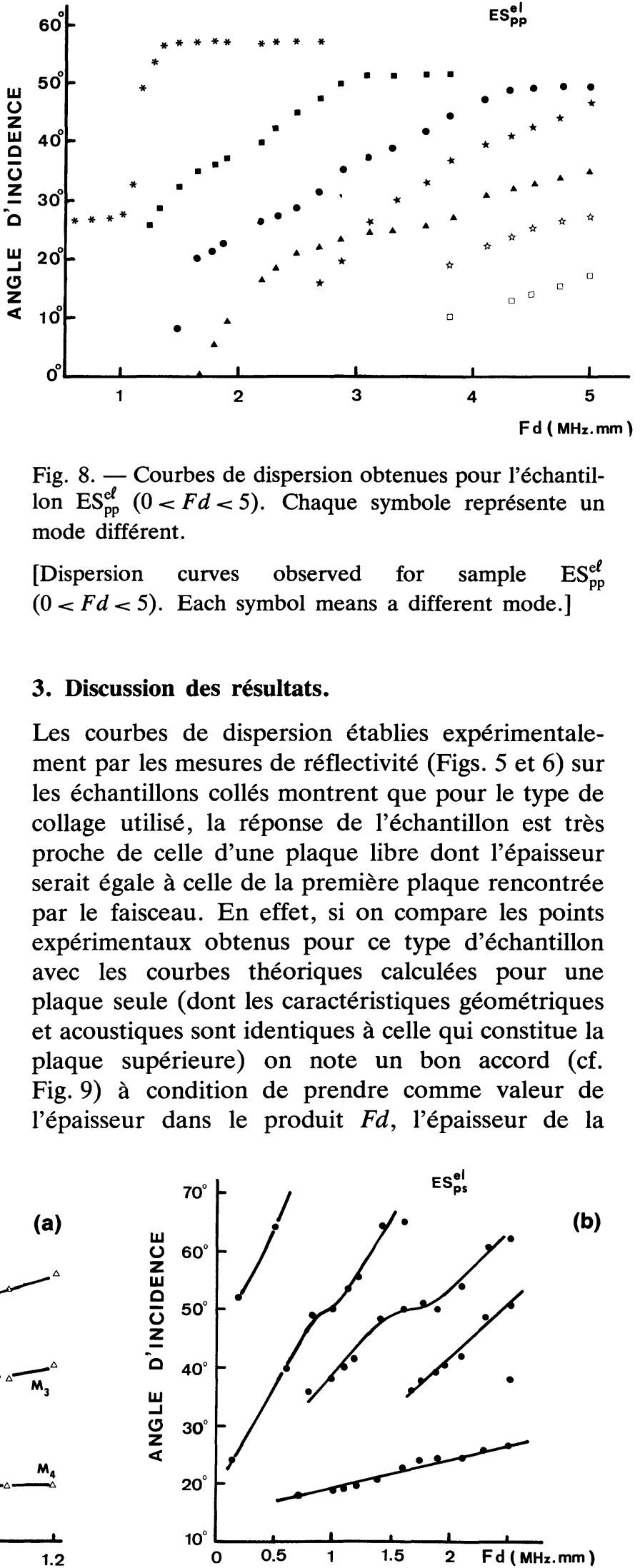

Fig. 8. - Courbes de dispersion obtenues pour l'échantillon $\operatorname{ES}_{\mathrm{pp}}^{\mathrm{el}}(0<F d<5)$. Chaque symbole représente un mode différent.

[Dispersion curves observed for sample $\mathrm{ES}_{\mathrm{pp}}^{\mathrm{e} \ell}$ $(0<F d<5)$. Each symbol means a different mode.]

\section{Discussion des résultats.}

Les courbes de dispersion établies expérimentalement par les mesures de réflectivité (Figs. 5 et 6) sur les échantillons collés montrent que pour le type de collage utilisé, la réponse de l'échantillon est très proche de celle d'une plaque libre dont l'épaisseur serait égale à celle de la première plaque rencontrée par le faisceau. En effet, si on compare les points expérimentaux obtenus pour ce type d'échantillon avec les courbes théoriques calculées pour une plaque seule (dont les caractéristiques géométriques et acoustiques sont identiques à celle qui constitue la plaque supérieure) on note un bon accord (cf. Fig. 9) à condition de prendre comme valeur de l'épaisseur dans le produit $F d$, l'épaisseur de la

a)

(b) 


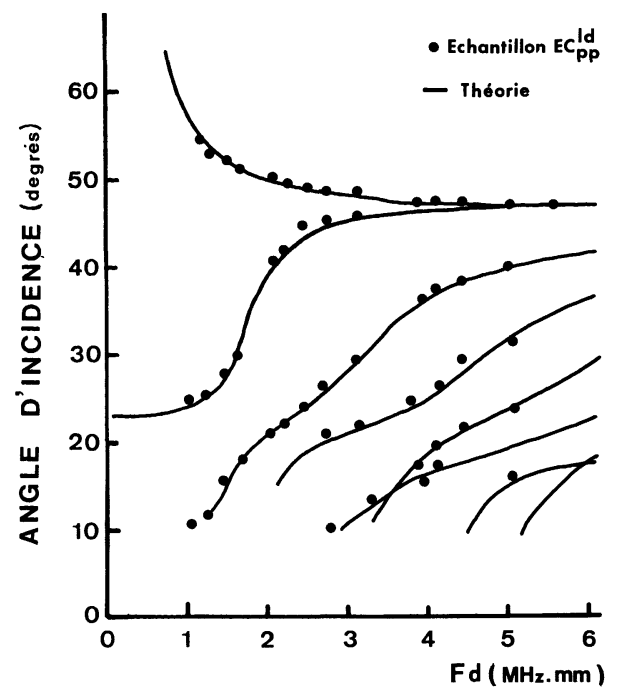

Fig. 9. - Etude comparative des courbes de dispersion obtenues pour l'échantillon $E C_{\mathrm{pp}}^{\ell_{\mathrm{d}}}$. (-) Courbes théoriques calculées pour une plaque libre. (•) Points expérimentaux de la figure 6a.

[Comparative study of dispersion curves for sample $\mathrm{EC}_{\mathrm{pp}}^{\ell_{\mathrm{d}}}$. (-) Theoretical curves for a free plate. (•) Experimentals points of the picture 6a.]

première plaque rencontrée par le faisceau acoustique incident. Sur la figure 9, les traits continus représentent les courbes de dispersion obtenues théoriquement à partir des équations des modes de Lamb (cf. [21]).

Ce résultat confirme que la colle utilisée n'agit pas comme un couplant rigide mais plutôt comme une plaque fluide ne transmettant pas d'onde de cisaillement. Les propriétés acoustiques du joint de colle (viscosité, coefficient de raideur, adhésion, ...) sont probablement responsables de ce comportement. Des expériences complémentaires menées sur des échantillons présentant une structure multicouche analogue, mais d'épaisseur de couplant différente $(100$ et $50 \mu \mathrm{m})$ ont confirmé les courbes de dispersion obtenues précédemment (Fig. 5). Ces mesures révèlent le peu d'influence de l'épaisseur du couplant sur les mesures en réflexion. Ces observations concernent tout particulièrement les modes $A_{0}$, $S_{0}$ et $A_{1}$ qui sont en général bien isolés et le type de collage décrit précédemment.

Une étude comparative portant sur les courbes de dispersion des modes a été faite avec des mesures effectuées en transmission dans le cas de l'échantillon $\mathrm{EC}_{\mathrm{pp}}^{\ell \mathrm{d}}$ (Fig. 10). Il est intéressant de noter que les résultats obtenus mettent cette fois en évidence la présence de courbes de dispersion relatives aux deux plaques constituant l'échantillon. A condition d'adapter l'échelle des abscisses $(F d)$ aux épaisseurs respectives des deux plaques métalliques traversées, on retrouve les courbes de dispersion propres à chacune des couches supposées libres. L'échelle

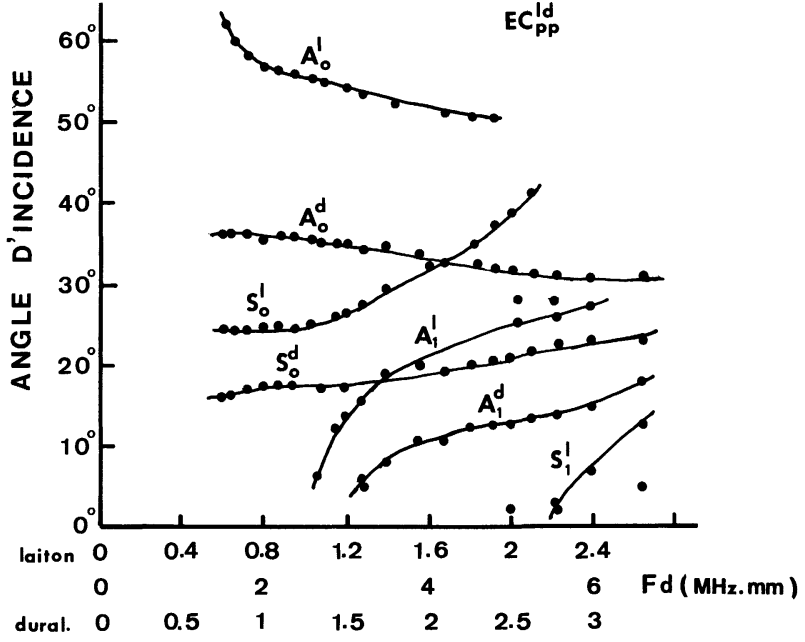

Fig. 10. - Courbes de dispersion obtenues par transmission pour l'échantillon $\mathrm{EC}_{\mathrm{pp}}^{\ell \mathrm{d}}$. Les indices supérieurs utilisés pour l'identification des modes se rapportent respectivement aux différentes plaques $(\ell$, laiton et $\mathrm{d}$, duraluminium).

[Dispersion curves for sample $\mathrm{EC}_{\mathrm{pp}}^{\ell \mathrm{d}}$ by transmission. The upper indexis used for identification of the modes correspond to the different plates $(\ell$, brass and $d$, duraluminum).]

intermédiaire (cf. Fig. 10) correspond au produit de la fréquence par l'épaisseur totale de l'échantillon.

Les courbes de dispersion obtenues dans le cas de l'échantillon comprenant une plaque d'étain soudée sur un substrat de laiton (Fig. 7) confirment le caractère «loading » du système - par opposition au caractère «stiffening». (Ces deux termes sont empruntés au vocabulaire anglo-saxon.) Ceci est dû au fait que la vitesse de l'onde de cisaillement dans la couche d'étain est inférieure à la vitesse de l'onde du même type dans le substrat (laiton) cf. table I. Sur les figures $7 a$ et $7 b$, nous avons relié les points expérimentaux entre eux pour chaque mode existant. Les différentes courbes de dispersion ont des comportements analogues à ceux prévus par les calculs théoriques publiés jusqu'à présent et correspondent suivant les auteurs aux modes $\mathbf{M}_{11}, \mathbf{M}_{21}$, $\mathbf{M}_{12}, \ldots$ [19], aux modes $\mathbf{M}_{0}, \mathbf{M}_{1}, \mathbf{M}_{2}, \ldots$ [12] ou aux ondes de Rayleigh, de Sezawa et de Rayleigh d'ordre supérieur [13, 14, 17]. Remarquons que pour ce type d'échantillon, aucune courbe de dispersion ne ressemble, comme dans le cas des plaques collées, à celles observées pour une plaque seule. L'onde de Sezawa n'est en fait que l'autre appellation du mode $M_{1}$. Cette onde, à partir d'une fréquence de coupure, doit rayonner dans le substrat $[14,15,22]$.

Il est intéressant aussi de remarquer que, bien que la théorie ne prévoit pas d'ondes d'interface rayonnant dans le milieu fluide au-delà de l'angle critique transverse, associé au substrat $[17,19]$, l'expérience, 
quant à elle, met en évidence des minima dans les variations du coefficient de réflexion. Ces creux peuvent s'expliquer par la génération d'ondes d'interface réémettant dans le substrat, provoquant ainsi une perte d'énergie en réflexion. Ces minima ne résultent donc pas d'un effet non spéculaire mais plutôt d'un effet de «pseudo-transmission » dans le milieu inférieur (substrat). Cette interprétation est confirmée par les profils des faisceaux réfléchis enregistrés à des angles qui correspondent respectivement à la génération d'une onde de Rayleigh (Fig. 11a) d'une réflexion spéculaire (Fig. 11b) et d'une pseudo-onde de Sezawa (Fig. 11c). On constate que seul le faisceau de la figure 11a est déformé justifiant ainsi l'existence d'un effet non spéculaire produit par la réémission, dans le milieu fluide, de l'onde de Rayleigh. Cette déformation n'apparaît pas dans le cas de la pseudo-onde de Sezawa puisque l'angle de génération est inférieur à $\theta_{\mathrm{T}}$.

En ce qui concerne l'échantillon $\mathrm{ES}_{\mathrm{pp}}^{\mathrm{e} \ell}$, les résultats expérimentaux représentés sur la figure 8 sont identi-

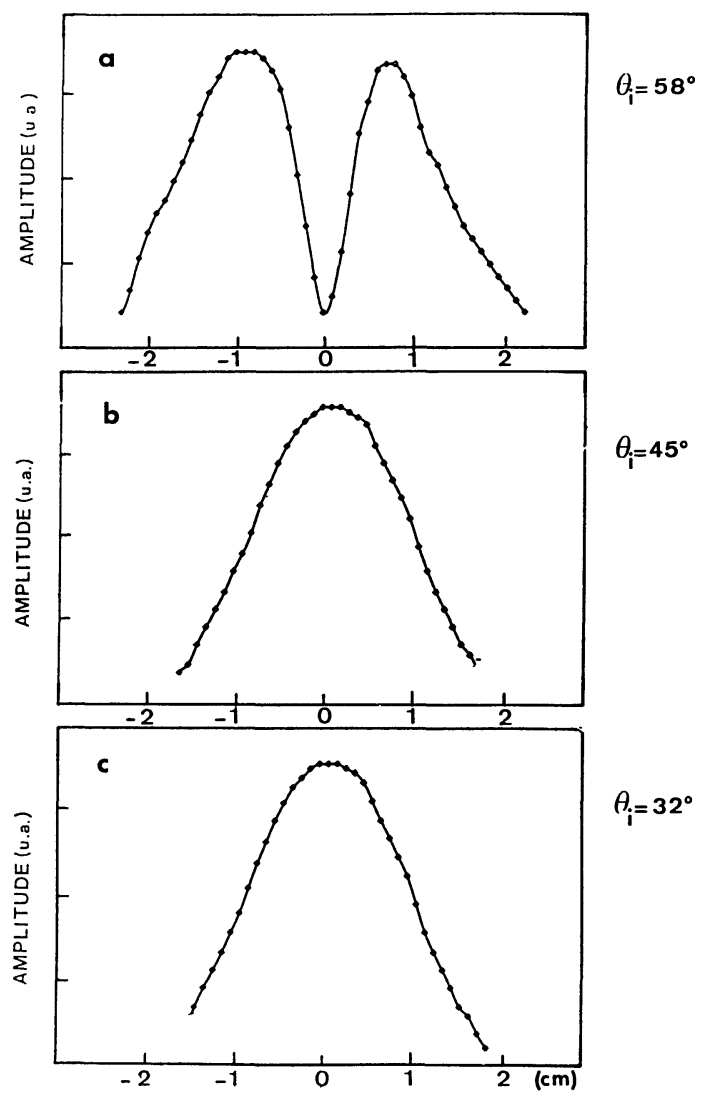

Fig. 11. - Profils des faisceaux réfléchis enregistrés perpendiculairement à l'axe moyen du faisceau réfléchi pour différents angles d'incidence à $F d=0,4 \mathrm{MHz} . \mathrm{mm}$.

[Reflected beam profiles at different incident angles. These profiles have been recorded perpendicularly to the reflected beam's axis at $F d=0.4 \mathrm{MHz} . \mathrm{mm}$.] ques, que les mesures soient réalisées en réflexion ou en transmission. Ces courbes ne sont caractéristiques ni de la plaque d'étain libre, ni de la plaque de laiton. Elles doivent être représentatives d'un système multicouche se comportant comme une couche unique équivalente dont les caractéristiques acoustiques restent à préciser, de même que les conditions aux limites. Ces résultats doivent être rapprochés des calculs théoriques effectués par Lloyd et Redwood [20] pour trois conditions différentes de contact entre deux plaques solides de matériaux différents : un contact parfait, un contact avec glissement et un contact par l'intermédiaire d'une couche de fluide.

\section{Conclusion.}

Cette étude menée en incidence oblique sur des systèmes multicouches et fondée sur des mesures de réflectivité a révélé qu'on ne peut traiter le collage entre deux plaques comme un couplage parfait puisque les deux plaques collées se comportent pratiquement comme une plaque libre de caractéristiques acoustiques et géométriques analogue à la première plaque rencontrée par le faisceau acoustique incident. Ces résultats confirment également que les modes présents aux faibles valeurs de $F d$ (donc aux grandes longueurs d'onde) sont plus sensibles aux caractéristiques géométriques et élastiques des différents milieux et donc plus intéressants à analyser en vue d'une caractérisation du couplage. D'autre part les expériences réalisées sur les échantillons soudés ont confirmé les calculs théoriques ainsi que le comportement des ondes d'interface en deçà et au-delà de l'angle critique transverse du substrat.

Les résultats expérimentaux rapportés dans cet article devraient apporter des éléments d'information pour l'élaboration des modèles théoriques et le choix des conditions aux limites toujours délicates à formuler.

Des études complémentaires doivent encore être faites pour analyser les comportements des échantillons collés du type $\mathrm{EC}_{\mathrm{pp}}$, en réflexion et en transmission, qui se sont révélés être très différents. Un prolongement de cette étude est aussi nécessaire pour permettre une meilleure compréhension de l'influence de l'épaisseur de la couche et de ses propriétés viscoélastiques sur les courbes de dispersion de la vitesse de phase.

\section{Remerciements.}

Cette étude a été supportée par la Direction des Recherches, Etudes et Techniques (D.R.E.T., Contrat 87-488). 


\section{Bibliographie}

[1] Bertoni, H. L. and TAmir, T., Appl. Phys. 2 (1973) 157-172.

[2] Neubauer, W. G., J. Appl. Phys. 44 (1973) 48-55.

[3] Becker, F. L. and Richardson, R. L., Physical Acoustics, Ed. W. P. Mason, Vol. IV, chapter 4 ; et J. Acoust. Soc. Am. 45 (1969) 793-794.

[4] N'goc, T. D. K. and MAYer, W. G., J. Acoust. Soc. Am. 67 (1980) 1149-1152.

[5] Breazeale, M. A., Adler, L. and Scott, G. W., J. Appl. Phys. 48 (1977) 530-537.

[6] N'GOC, T. D. K. and MAYER, W. G., IEEE Trans. Sonics Ultrasonics SU 27 (1980) 229-236.

[7] Claeys, J. M., Leroy, O. J., N'GOC, T. D. K. and MAYer, W. G., Acoust. Lett. 5 (1981) 48-54.

[8] De Billy, M. and Quentin, G., Ultrasonics (1984) pp. 249-251.

[9] Nayfeh, A. H., Chimenti, D. E., Adler, L. and Crane, R. L., J. Appl. Phys. 52 (1981) 49854994.

[10] Chimenti, D. E., Nayfeh, A. H. and Butler, D. L., J. Appl. Phys. 53 (1982) 170-176.

[11] NAyfeh, A. H. and Chimenti, D. E., J. Acoust. Soc. Am. 75 (1984) 1360-1368.
[12] Hattunen, M. and Luukkala, M., Appl. Phys. 2 (1973) $257-263$

[13] Luukkala, M. and Hattunen, M., Appl. Phys. Lett 19 (1971) 56-57.

[14] Tsukahara, Y., Nakaso, N., Ohira, K., Saito, M., KUSLIBIKI, J. and CHUbACHI, N., Ult. Int. 87 Conf. Proc. (1987) pp. 465-470.

[15] Tsukahara, Y., Takeuchi, E. and Hayashi, E., Ultra. Sympo. (1984) 992-996.

[16] Bogy, D. B. and Gracewski, S. M., J. Acoust. Soc. Am. 74 (1983) 591-594.

[17] Jackins, P. D. and Gaunaurd, G. C., J. Acoust. Soc. Am. 80 (1986) 1762-1776.

[18] Farnell, G. W. and Adler, E. L., Physical Acoustics (Academic Press N.Y. et Londres) Vol. IX (1972) 35-125.

[19] Pilarski, A., Arch. Acoust. 7 (1982) 61-70.

[20] Lloyd, P. and Redwood, M., Acustica 16 (1965) 224-232.

[21] BREKHOVSKIKH, Waves in Layered Media (Academic Press. New York) 1960.

[22] SEZAWA, K., KANAI, K., Bull. Earthquake Res. Inst. 13 (1935) 238. 